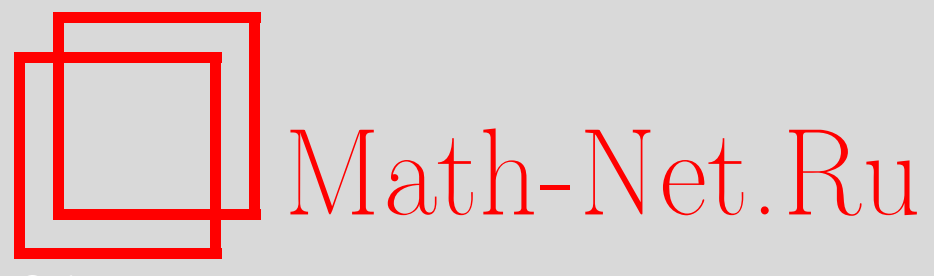

Ф. С. Мышаков, Аналог теоремы Валирона-Гольдберга при ограничении на усредненную считающую функцию множества корней, Матем. заметки, 2014, том 96, выпуск $5,794-798$

DOI: https://doi.org/10.4213/mzm10538

Использование Общероссийского математического портала Math-Net.Ru подразумевает, что вы прочитали и согласны с пользовательским соглашением http: //www . mathnet.ru/rus/agreement

Параметры загрузки:

IP: 52.6 .47 .48

26 апреля 2023 г., 16:11:00

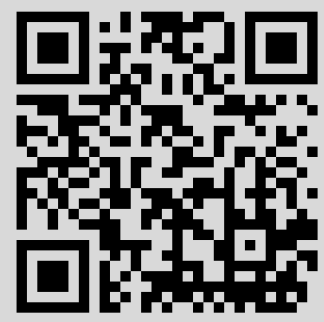




\section{Аналог теоремы Валирона-Гольдберга при ограничении на усредненную считающую функцию множества корней}

\section{Ф. С. Мышаков}

1. Введение. Сто лет назад Валирон [1] рассмотрел следующую задачу. Имеется целая функция $f$ конечного нецелого порядка. Задана “достаточно регулярная" $n_{f}(R)-$ количество корней $f$ в круге $|z| \leqslant R$. Требуется найти возможно лучшую асимптотическую оценку сверху величины $\ln M(f, R)$, где

$$
M(f, R)=\max _{|z| \leqslant R}|f(z)| .
$$

Эта задача в "первом приближении" была решена самим Валироном в [1], а позже Гольдберг [2] дал строгое доказательство этого результата и установил его неулучшаемость. Дальнейшее продвижение в этой тематике было недавно получено Поповым [3]. В данной статье решена аналогичная задача, в которой задана мажоранта усредненной считающей функции множества ненулевых корней $f(z)$ :

$$
N_{f}(R)=\int_{0}^{R}\left(\frac{n_{f}^{0}(x)}{x}\right) d x,
$$

где $n_{f}^{0}(x)=n_{f}(x)-m, m-$ кратность корня $f(z)$ в точке $z=0$.

Величина $N_{f}(R)$ более тесно, чем $n_{f}(R)$, связана с асимптотическим поведением логарифма модуля функции $f$. Согласно формуле Йенсена

$$
N_{f}(R)=\frac{1}{2 \pi} \int_{0}^{2 \pi} \ln \left|f\left(R e^{i \varphi}\right)\right| d \varphi-m \ln R-\ln \left|\frac{f^{(m)}(0)}{m !}\right|
$$

при больших $R$ с точностью $O(\ln R)$ совпадает со средним логарифма модуля $f(z)$ на окружности $|z|=R$. В случае $f(0)=1$ указанное среднее есть в точности $N_{f}(R)$. Поэтому задача оценки сверху $\ln M(f, R)$ через $N_{f}(R)$ фактически является задачей оценки сверху максимума функции $\ln \left|f\left(R e^{i \varphi}\right)\right|$ на отрезке $0 \leqslant \varphi \leqslant 2 \pi$ через ее среднее на этом отрезке и выглядит нереальной. Но специфика асимптотического поведения логарифма модуля целой функции нецелого порядка такова, что оценка сверху $\ln M(f, R)$ (с некоторым не зависящим от $R$ множителем) через достаточно регулярную мажоранту функции $N_{f}(R)$ при всех достаточно больших $R$ уже оказывается возможной.

Напомним несколько определений и теорему о факторизации целой функции конечного нецелого порядка [4; (гл. 1)].

Уточненным порядком называется произвольная функция $\rho(r)$, определенная и дифференцируемая на луче $R_{0}<r<+\infty$ (число $R_{0}$ свое для каждой такой функции) и обладающая следующими свойствами:

$$
\lim _{r \rightarrow+\infty} \rho(r)=\rho, \quad 0<\rho<+\infty, \quad \lim _{r \rightarrow+\infty} \rho^{\prime}(r) r \ln r=0 .
$$

Верхней плотностъю множества корней функции $f$ относительно уточненного порядка $\rho(r)$ называется величина

$$
D_{\rho(r)}(f)=\limsup _{r \rightarrow+\infty} r^{-\rho(r)} n_{f}(r) .
$$

DOI: $10.4213 / \mathrm{mzm} 10538$ 
Усредненной верхней плотностъю множества корней функции $f$ относительно уточненного порядка $\rho(r)$ называется величина

$$
D_{\rho(r)}^{*}(f)=\limsup _{r \rightarrow+\infty} r^{-\rho(r)} N_{f}(r) .
$$

Известно неулучшаемое с обеих сторон двойное неравенство [4]

$$
\rho D_{\rho(r)}^{*} \leqslant D_{\rho(r)} \leqslant e \rho D_{\rho(r)}^{*}, \quad \text { где } \quad \rho=\lim _{r \rightarrow+\infty} \rho(r) .
$$

Как известно, любая целая функция $f$ нецелого порядка имеет бесконечно много корней; через $\Lambda_{f}=\left\{\lambda_{n}\right\}_{n \in \mathbb{N}}$ обозначим множество ее корней, отличных от точки $z=0$, упорядоченных по неубыванию модулей. Каждый корень стоит в последовательности $\Lambda_{f}$ столько раз, какова его кратность. Через $f_{\Lambda}$ обозначим каноническое произведение по ненулевым корням целой функции $f$ порядка $\rho$ :

$$
\begin{gathered}
f_{\Lambda}(z)=\prod_{n=1}^{\infty} E_{p}\left(\frac{z}{\lambda_{n}}\right), \quad \text { где } p=[\rho], \\
E_{0}(w)=1-w, \quad E_{p}(w)=(1-w) \exp \left(\sum_{k=1}^{p} \frac{w^{k}}{k}\right), \quad p \in \mathbb{N} .
\end{gathered}
$$

Согласно теореме Адамара сама целая функция $f$ допускает представление

$$
f(z)=z^{m} \exp (\mathcal{P}(z)) f_{\Lambda}(z),
$$

где $m$ - кратность нуля в точке $z=0$ (множитель $z^{m}$ отсутствует, если $\left.f(0) \neq 0\right), \mathcal{P}-$ некоторый многочлен степени $\leqslant p$.

Важную роль в статье играет функция $\mathcal{M}_{p}(r)=\ln \max _{|w|=r}\left|E_{p}(w)\right|$, исследованная Данжуа [5]. Валирон [1] ввел функцию

$$
S(\rho)=\int_{0}^{+\infty} r^{-\rho} d \mathcal{M}_{p}(r)=\rho \int_{0}^{+\infty} r^{-\rho-1} \mathcal{M}_{p}(r) d r, \quad \rho \in(0,+\infty) \backslash \mathbb{N}, \quad p=[\rho],
$$

и доказал, опираясь на теорему А. Данжуа [5] о поведении функции $d \mathcal{M}_{p}(r) / d r$, сходимость этого несобственного интеграла.

Сформулируем теорему Валирона-Гольдберга.

Tеорема A [1], [2]. Пусть $\rho(r)$ - произволъный уточненный порядок, $\lim _{r \rightarrow+\infty} \rho(r)=$ $\rho \in(0,+\infty) \backslash \mathbb{N}, f$ - произвольная целая функиия порядка $\rho$, множество корней которой имеет конечную верхнюю плотность относительно этого уточненного порядка. Тогда $f$ имеет конечный тип при порядке $\rho(r)$ и справедливо неравенство

$$
\sigma_{\rho(r)}(f) \leqslant S(\rho) D_{\rho(r)}(f) .
$$

С другой стороны, существует функция $f$, имеющая положительный тип при порядке $\rho(r)$, для которой неравенство (7) обращается в равенство.

Этот результат перенесен автором на случай, когда задана усредненная верхняя плотность множества корней.

Теорема 1. В условиях теоремы А справедливо неравенство

$$
\sigma_{\rho(r)}(f) \leqslant \rho S(\rho) D_{\rho(r)}^{*}(f) .
$$

и существует функция $f$, для которой неравенство (8) обращается в равенство. 
Оценка сверху (8) не следует из (7), поскольку согласно (3) $D$ оценивается через $\rho D^{*}$ не сверху, а снизу. Но зато из $(3),(7),(8)$ видно, что если для некоторой функции $f$ неравенство (7) обращается в равенство, то для этой же функции обращается в равенство и неравенство (8).

Неравенство (8) было известно ранее (см. [6; гл. 5, §3], [7]) при ограничении $0<\rho<1$ и лишь в случае $\rho(r) \equiv r$. При этих значениях $\rho$ функция $S(\rho)$ допускает простое выражение: $S(\rho)=\pi \operatorname{cosec}(\pi \rho)$, но при $\rho>1$ функция $S(\rho)$ не элементарна. В общем случае теорема 1 , по-видимому, является новой.

В статье доказывается более детальный результат. Предполагается, что усредненная считающая функция множества корней допускает оценку сверху

$$
N_{f}(R) \leqslant r^{\rho(r)}+O\left(r^{a}\right), \quad 0 \leqslant a<\rho .
$$

и из нее выводится асимптотическая оценка сверху $\ln M(f, r)$ с двумя неулучшаемыми слагаемыми.

2. Основные результаты. Сначала рассмотрим уточненные порядки $\rho(r)$, отличающиеся "правильностью" поведения при $r \rightarrow+\infty$. Из определения (1) следует, что если $\rho(r)-$ произвольный уточненный порядок, $\lim _{r \rightarrow+\infty} \rho(r)=\rho$, то функция $l(r)=r^{\rho(r)-\rho}$ является медленно меняющейся [8] и справедливо предельное соотношение

$$
\lim _{r \rightarrow+\infty} \frac{r l^{\prime}(r)}{l(r)}=0
$$

Потребуем, чтобы уточненный порядок удовлетворял следующим условиям:

1) функция $l(r)$ монотонна,

2) функция $w_{l}(r)=r l^{\prime}(r) / l(r)$ также монотонна (она может быть либо положительной, либо отрицательной) и медленно меняется на бесконечности.

Эти условия выполняются, в частности для функций

$$
\begin{array}{ll}
l(r)=\exp \left(a(\ln r)^{b}\right), & a, b \in \mathbb{R}, \quad b<1, \\
l(r)=(\ln r)^{a}(\ln \ln r)^{b}, & a, b \in \mathbb{R} .
\end{array}
$$

Теорема 2. Пусть задан уточненный порядок $\rho(r), \lim _{r \rightarrow+\infty} \rho(r)=\rho \in(0,+\infty) \backslash \mathbb{N}$, удовлетворяющий сформулированным выше условиям 1), 2). Если для усредненной считающей функции множества корней целой функиии $f$ порядка $\rho$ выполняется асимптотическая оченка сверху (9), то

$$
\ln M(f, R) \leqslant R^{\rho(R)}\left(\rho S(\rho)+\left(\rho S^{\prime}(\rho)+S(\rho)\right) w_{l}(R)+o\left(w_{l}(R)\right)\right), \quad R \rightarrow+\infty .
$$

Если для усредненной считающей функиии $N(r)$ возрастающей последовательности положительных чисел $\left\{r_{n}\right\}_{n \in \mathbb{N}}, \lim _{n \rightarrow+\infty} r_{n}=+\infty$, выполняется двусторонняя асимптотическая оченка

$$
r^{\rho(r)}+O\left(r^{a}\right) \leqslant N(r)=O\left(r^{\tau}\right), \quad r \rightarrow+\infty,
$$

в которой $\tau<[\rho]+1$, то найдутся числовые последовательности $\varphi_{n} \in[\pi, \pi)$ u $R_{k} \rightarrow+\infty$ такие, что для логарифма максимума модуля на окружностях $|z|=R_{k}$ любой целой функции $F$, множество всех корней которой есть $\left\{r_{n} e^{i \varphi_{n}}\right\}_{n \in \mathbb{N}}$, справедлива асимптотическая оценка снизу

$$
\ln M\left(F, R_{k}\right) \geqslant R_{k}^{\rho\left(R_{k}\right)}\left(\rho S(\rho)+\left(\rho S^{\prime}(\rho)+S(\rho)\right) w_{l}\left(R_{k}\right)+o\left(w_{l}\left(R_{k}\right)\right)\right), \quad k \rightarrow+\infty .
$$


Теперь рассмотрим произвольный уточненный порядок $\rho(r)$. В этом общем случае от функции $l$ ничего не требуется, кроме выполнения предельного соотношения (10). Возьмем произвольную положительную невозрастающую на некотором луче $\left(r_{0},+\infty\right)$ функцию $w(r), \lim _{r \rightarrow+\infty} w(r)=0$. Введем класс $\mathcal{L}(w)$, состоящий по определению из всех таких медленно меняющихся и дифференцируемых на $\left(r_{0},+\infty\right)$ функций $l$, что верно неравенство $\left|w_{l}(r)\right| \leqslant w(r)$.

ЗАмечАниЕ. Из определения класса $\mathcal{L}(w)$ следует, что каков бы ни был уточненный порядок $\rho(r) \rightarrow+\infty$, порождаемая им по формуле $l(r)=r^{\rho(r)-\rho}$ медленно меняющаяся функция принадлежит одному из классов $\mathcal{L}(w)$, причем функцию $w$ можно взять медленно меняющейся.

Обозначим

$$
\begin{aligned}
& S_{1}(\rho)=\int_{0}^{+\infty}|\ln r| r^{-\rho} d \mathcal{M}_{p}(r), \\
& S_{0}(\rho)=\int_{0}^{1} r^{-\rho} d \mathcal{M}_{p}(r)-\int_{1}^{+\infty} r^{-\rho} d \mathcal{M}_{p}(r), \quad p=[\rho] .
\end{aligned}
$$

Теорема 3. Пусть $w:\left(r_{0},+\infty\right) \rightarrow(0,+\infty)$ - произвольная невозрастающая медленно меняющаяся на бесконечности функиия, $\lim _{r \rightarrow+\infty} w(r)=0, \rho(r)$ - произвольный уточненный порядок такой, что $\lim _{r \rightarrow+\infty} \rho(r)=\rho \in(0,+\infty) / \mathbb{N}, l(r)=r^{\rho(r)-\rho} \in \mathcal{L}(w)$. Тогда для логарифма максимума модуля иелой функции порядка $\rho$, усредненная считающая функиия корней которой удовлетворяет ограничению (9), справедлива асимптотическая оценка сверху

$$
\ln M(f, R) \leqslant R^{\rho(R)}\left(\rho S(\rho)+\left(\rho S_{1}(\rho)+S_{0}(\rho)\right) w(R)+o(w(R))\right), \quad R \rightarrow+\infty .
$$

Изложим идеи доказательства теорем 2, 3 (теорема 1 несложно выводится из теоремы 3). Из представления видно, что теоремы 2,3 достаточно доказать для канонических произведений (4). Имеем

$$
\ln M\left(f_{\Lambda}, R\right) \leqslant \sum_{n=1}^{\infty} \mathcal{M}_{p}\left(\frac{R}{\left|\lambda_{n}\right|}\right),
$$

а в [7] доказано, что всегда можно так подобрать аргументы корней $\lambda_{n}$, что на некоторой последовательности окружностей $|z|=R_{k}$ разность величин

$$
\ln M\left(f_{\Lambda}, R_{k}\right) \quad \text { и } \quad \sum_{n=1}^{\infty} \mathcal{M}_{p}\left(\frac{R_{k}}{\left|\lambda_{n}\right|}\right)
$$

будет $R_{k}^{\max (p, a)+\varepsilon}$ для любого $\varepsilon>0$ при $k \rightarrow \infty$.

После этого остается только найти неулучшаемую по порядку асимптотическую оценку сверху суммы

$$
\begin{aligned}
\sum_{n=1}^{\infty} \mathcal{M}_{p}\left(\frac{r}{\left|\lambda_{n}\right|}\right) & =\int_{0}^{+\infty} \mathcal{M}_{p}\left(\frac{r}{x}\right) d n_{\Lambda}(x)=\int_{0}^{+\infty} n_{\Lambda}(x) d \mathcal{M}_{p}\left(\frac{r}{x}\right) \\
& =\int_{0}^{+\infty} n_{\Lambda}\left(\frac{R}{t}\right) \mathcal{M}_{p}^{\prime}(t) d t=\int_{0}^{+\infty} N_{\Lambda}\left(\frac{R}{t}\right) d\left(t \mathcal{M}_{p}^{\prime}(t)\right) .
\end{aligned}
$$

Автором доказано возрастание функции $t \mathcal{M}_{p}^{\prime}(t)$. Вследствие этого из (9) находим

$$
\int_{0}^{+\infty} N_{\Lambda}\left(\frac{R}{t}\right) d\left(t \mathcal{M}_{p}^{\prime}(t)\right) \leqslant \int_{0}^{+\infty}\left(\frac{R}{t}\right)^{\rho(R / t)} d\left(t \mathcal{M}_{p}^{\prime}(t)\right)+O\left(\int_{0}^{+\infty}\left(\frac{R}{t}\right)^{a} d\left(t \mathcal{M}_{p}^{\prime}(t)\right)\right) .
$$


Заключительная часть доказательства состоит в исследовании асимптотического поведения интеграла

$$
\int_{0}^{+\infty}\left(\frac{R}{t}\right)^{\rho} l\left(\frac{R}{t}\right) d\left(t \mathcal{M}_{p}^{\prime}(t)\right)
$$

Главный член его асимптотики выделяется сравнительно просто, а самая большая трудность в работе - нахождение второго члена асимптотики интеграла (16). Это удается сделать, если функция $l$ отличается "регулярным" поведением. В общем случае получена асимптотическая оценка сверху второго члена на классах медленно меняющихся функций $l$ и доказана точность этой оценки по порядку.

\section{СПИСОК ЦИТИРОВАННОЙ ЛИТЕРАТУРЫ}

[1] G. Valiron, "Sur les fonctions entières d'ordre nul et d'ordre fini et en particulier les fonctions à correspondance régulière", Ann. Fac. Sci. Toulouse Sci. Math. Sci. Phys. (3), 5 (1913), 117-257. [2] А. А. Гольдберг, Матем. сб., 58:3 (1962), 289-334. [3] А. Ю. Попов, Матем. сб., 204:5 (2013), 67-108. [4] Б. Я. Левин, Распределение корней иелых функиий, Гостехиздат, M., 1956. [5] А. Denjoy, J. Math. (6), 6 (1910), 1-136. [6] А. А. Гольдберг, И. В. Островский, Распределение значений мероморфных функиий, Наука, М., 1970. [7] Б. Н. Хабибуллин, Матем. сб., 200:2 (2009), 129-158. [8] Е. Сенета, Правильно меняющиеся функиии, Наука, M., 1985. 